\title{
Who Benefits Most from THC:CBD Spray? Learning from Clinical Experience
}

\author{
Jürgen Koehler \\ Marianne-Strauss-Klinik, Behandlungszentrum Kempfenhausen, Berg, Germany
}

\section{Key Words}

THC · CBD · Cannabinoids · Multiple sclerosis · Spasticity ·

Cases $\cdot$ Daily practice

\begin{abstract}
Patients with multiple sclerosis (MS) represent a diverse and heterogeneous population varying in terms of disease type, its severity and variable progression/time-course, and with regard to the wide range of presenting symptoms. Consequently, detailed experience with individual patients is important to provide examples of therapy to specific patient types. In this article, real-life data from clinical practice showing specific aspects relating to use of 9-delta-tetrahydocannabinol and cannabidiol (THC:CBD) oromucosal spray (Sativex ${ }^{\circledR}$ ) in patients with moderate to severe spasticity resistant to usual therapy will be presented. Three common clinical scenarios will be considered: MS patients with resistance to usual spasticity therapies; patients with impairment in MS spasticity symptoms; MS patients with relevant impairment in quality of life/activities of daily living (QoL/ADL). These case reports highlight the diverse nature of the MS spasticity population and they show the possible usefulness of THC:CBD oromucosal spray in individual patients with moderate to severe spasticity resistant to existing therapies, within the frame of use approved after large clinical trial results. Perhaps the most important finding is the possibility of
\end{abstract}

obtaining relevant improvements in QoL/ADL in some patients with resistant MS spasticity, allowing them to engage back in physical and social activities.

(c) 2014 S. Karger AG, Basel

\section{Introduction}

There is a wealth of data from Phase III clinical trials supporting the efficacy and safety of THC:CBD oromucosal spray (Sativex ${ }^{\circledR}$ ), a novel cannabinoid-based medicine comprising a 1:1 mixture of 9-delta-tetrahydocannabinol (THC) and cannabidiol (CBD) [1-7] in patients with multiple sclerosis (MS)-related spasticity. This has been further supplemented by a growing database of realworld experience comprising thousands of MS patients [8-10; see Rekand, this issue]. Given the heterogeneous nature of the MS population in terms of type of disease, its variable progression/time-course and the wide variation in symptoms and their severity, detailed experience with individual patients is important to illustrate therapy possibilities to specific patient types.

At the Marianne-Strauss-Klinik in Berg, Germany we have treated over 200 patients with resistant MS spasticity with THC:CBD oromucosal spray since September 2011 (fig. 1). In this article findings are presented for three clinical scenarios (two particular cases selected for each

\section{KARGER}

E-Mail karger@karger.com

www.karger.com/ene
C 2014 S. Karger AG, Basel

0014-3022/14/0717-0010\$39.50/0
Jürgen Koehler

Marianne-Strauss-Klinik, Behandlungszentrum Kempfenhausen

Milchberg 21

DE-82335 Berg (Germany)

E-Mail Juergen.koehler@ms-klinik.de 
Fig. 1. Clinical experience with THC:CBD oromucosal spray in the Marianne Strauss Klinik (MSK, Berg, Germany).

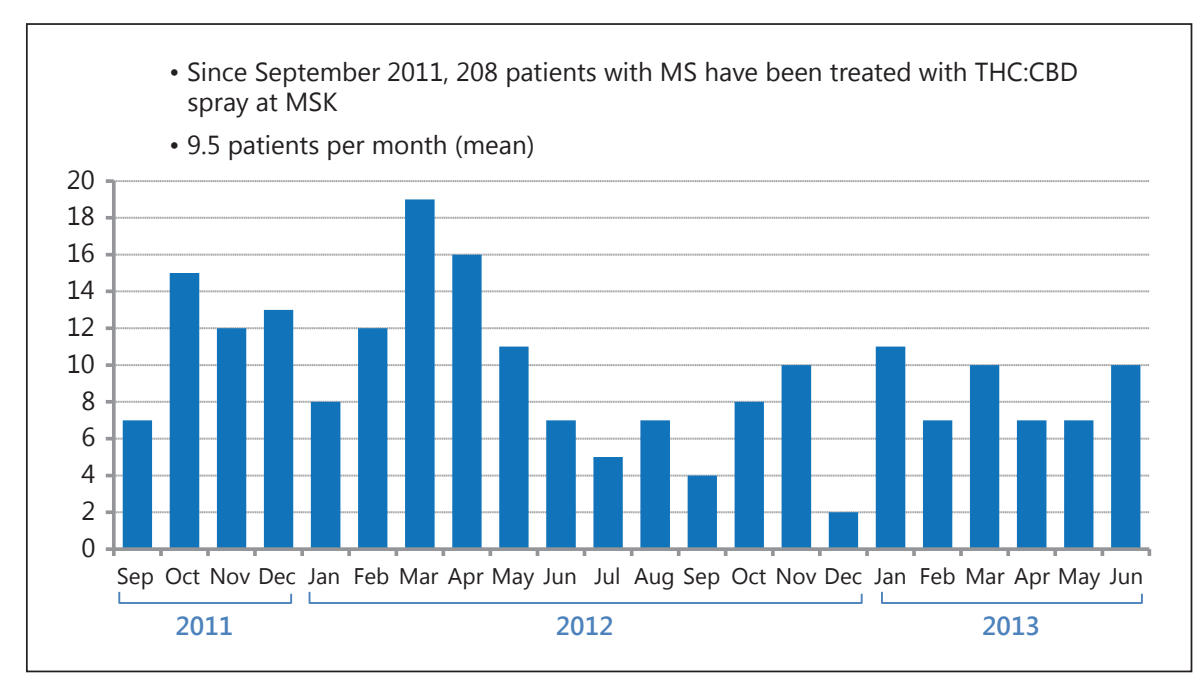

scenario) representing specific and typical clinical situations observed in everyday practice. Details of the patient's history prior to the introduction of THC:CBD oromucosal spray, the effectiveness of the new therapy and information regarding any changes made to optimize symptom control and manage adverse effects will be outlined. Details of two of these three clinical scenarios, as chosen by the audience, were presented at the ECTRIMS 2013 congress satellite symposium in Copenhagen. The three clinical scenarios were:

- MS patients with resistance to spasticity therapies

- Patients with impairment in their MS spasticity associated symptoms

- MS patients with relevant impairment in their quality of life/activities of daily living (QoL/ADL).

Systematic reviews describe limited evidence to support the efficacy of existing first-line therapies such as baclofen, tizanidine and gabapentin in patients with MS spasticity [11]. The German MOVE1 study demonstrated that $40-60 \%$ of physicians were dissatisfied with existing therapies for moderate to severe MS spasticity [12]. Thus, there appears to be a clear unmet need for better treatments to help physicians manage their patients with resistant moderate to severe MS spasticity.

\section{Clinical Scenario 1: MS Patients with Resistance to Spasticity Therapies}

\section{Case 1: Patient Details}

This case involved a 66-year-old female with secondary progressive MS, and an EDSS of 6.0. In 1979 the first incidence of MS (retrobulbar neuritis in left eye) was doc- umented. Since 2001 there has been a progressive decrease in walking ability and in 2009 the patient developed extreme temporary spasticity in the legs. Regular antispasticity medications such as baclofen, tizanidine and gabapentin were ineffective. MRI revealed pathological changes in the cervical segment of the spinal cord (fig. 2).

\section{Initial Treatment}

Intravenous corticosteroids (1,000 mg every 3 months) were insufficiently effective. Similarly, intrathecal triamcinolone was insufficiently effective. A baclofen pump was implanted and decreased the spasticity in the legs; however increased attacks of flexor spasticity occurred with this treatment.

\section{Addition of THC:CBD and Clinical Outcome}

Before THC:CBD oromucosal spray was started the patient remained on the baclofen pump $(225 \mu \mathrm{g} /$ day $)$ and could walk $252 \mathrm{~m}$ in $14 \mathrm{~min}$ with the use of a walking frame (EDSS 6.0), but still had attacks of flexor spasticity.

At a follow-up visit in June 2013 THC:CBD oromucosal spray was started at a dosage of one spray at night and increased up to a dosage of 3 sprays/day (morning, afternoon and evening). This specific add-on antispastic treatment has reduced the number of spasticity attacks significantly (NRS decreased from $5 / 10$ to $2 / 10$ ). Walking distance has been increased to $395 \mathrm{~m}$ in $20 \mathrm{~min}$.

\section{Case 2: Patient Details}

The second case involves a 45-year-old male with relapsing-remitting MS, and an EDSS of 6.0. In 1998 the first incidence of MS (ataxia, weakness, and sensory dis- 
Fig. 2. Case 1: MRI of the cervical segment of the spinal cord of a 66-year-old female with secondary progressive MS.

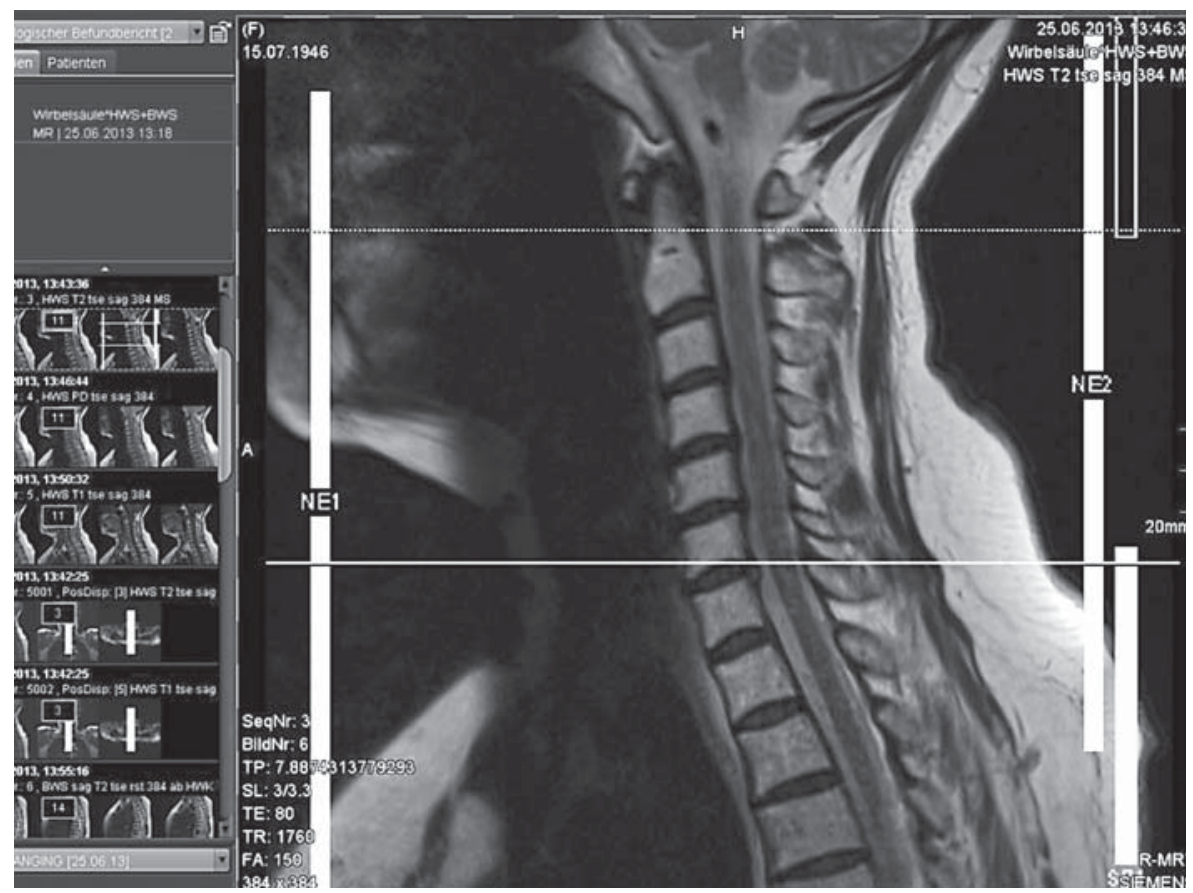

turbances of both legs) occurred. Between 2009 and 2011 the patient received interferon beta- $1 b$, and this was changed to fingolimod in October 2011 as a result of further relapses. The patient developed a severe recurrent equinovarus position of the right foot at rest and after tactile stimuli (a type of flexor spasticity), and the walking distance was $40 \mathrm{~m}$ in $1 \mathrm{~min}$ without a device (100 $\mathrm{m}$ with a walking frame). No antispastic medication was prescribed because of poor tolerability.

\section{Initial Treatment}

The patient was started on low dose baclofen but could not tolerate a dose $>10 \mathrm{mg} /$ day. Gabapentin and pregabalin was then tried, but these were also ineffective at dosages which the patient could tolerate without side effects.

\section{Addition of THC:CBD Oromucosal Spray and Clinical}

Outcome

THC:CBD oromucosal spray was initiated at a dosage of 2 sprays/day (morning and evening), in combination with baclofen $10 \mathrm{mg} / \mathrm{day}$, and this regimen significantly reduced the leg spasticity (NRS decreased from $8 / 10$ to 0/10). At a follow-up visit in June 2013 (after 148 days' treatment) the patient's condition was stable and he was receiving THC:CBD oromucosal spray 5 sprays/day (2 in the morning and evening and one in the afternoon) and he has stopped taking baclofen. The recurrent equinovarus position of the right foot which was evident prior to antispasticity therapy was markedly improved by THC:CBD (fig. 3) and the walking distance had increased to $170 \mathrm{~m}$ in $3 \mathrm{~min}$ without a device.

\section{Clinical Scenario 2: MS Patients with Impairment in MS Spasticity Associated Symptoms}

\section{Case 3: Patient Details}

The third case involved a 49-year-old male with secondary progressive MS, and an EDSS of 6.5. In 1997 the first incidence of MS (sensory disturbances) occurred. From 2002 onwards there has been progressive weakness in the arms and legs and since 2012 there has been an increasing incidence of painful spasticity at night. The patient had paresis of lower extremities with reduced muscle strength (2/5-3/5) and was able to walk $118 \mathrm{~m}$ in $6 \mathrm{~min}$ with a walking frame. He was receiving baclofen $10 \mathrm{mg}$ three times a day.

\section{Initial Treatment}

Increasing the baclofen dosage increased the weakness in the legs. 
Fig. 3. Case 2: Recurrent equinovarus position of the right foot at rest before and after treatment with THC:CBD oromucosal spray.

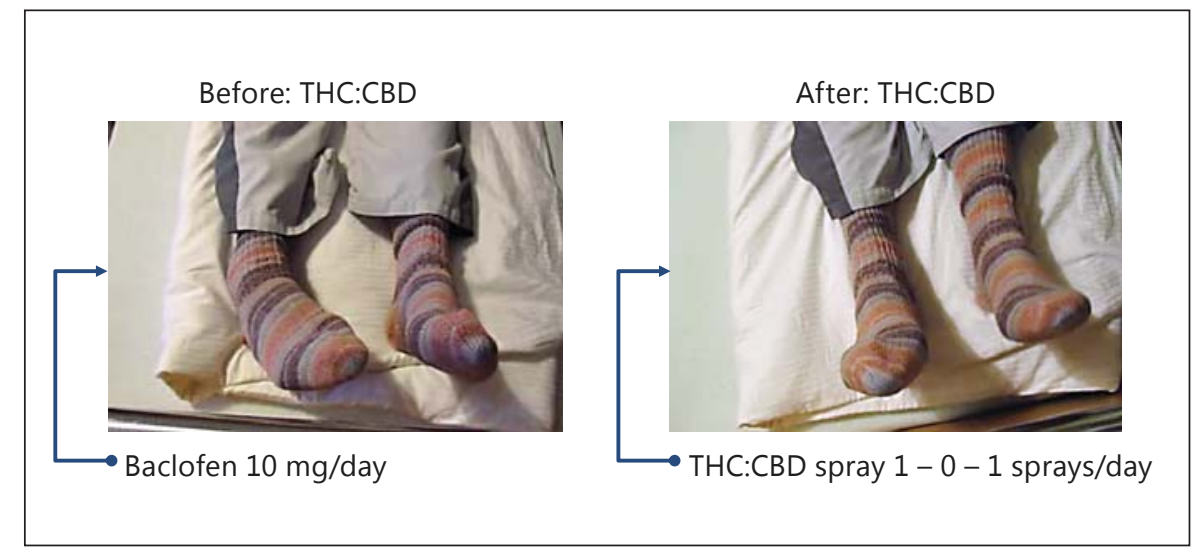

Addition of THC:CBD Oromucosal Spray and Clinical Outcome

THC:CBD oromucosal spray 6 sprays/day was started and there was an immediate improvement in hand function. Furthermore, there were no signs of weakness and both spasticity and pain were decreased (NRS 8/10 to 4/10). At a follow-up in June 2013 (344 days treatment) the patient had maintained stable improvement in NRS scores for pain and spasticity and lower limb paresis with muscle strength of 2/5-3/5. Treatment comprised THC:CBD oromucosal spray 6 sprays/day and baclofen $10 \mathrm{mg} /$ day. The walking distance was stable over at least one year and he could manage $100 \mathrm{~m}$ in 3 min with a walking frame compared with $118 \mathrm{~m}$ in $6 \mathrm{~min}$ before THC:CBD treatment.

\section{Case 4: Patient Details}

The fourth case involved a 53-year-old female with secondary progressive MS, and an EDSS of 8.0. In 1998 the first incidence of MS (diplopic images) occurred. From 2010 onwards there has been progressive weakness in the arms and legs, and since 2011 there has been an increasing incidence of painful spasticity attacks at night. The patient had paresis of lower extremities with muscle strength $2 / 5-3 / 5$ and was unable to walk due to recurrent flexor spasticity. She was receiving baclofen $(10 \mathrm{mg}$ in the morning and $15 \mathrm{mg}$ at night) and pregabalin (100 $\mathrm{mg}$ in the morning, $75 \mathrm{mg}$ in the afternoon and $125 \mathrm{mg}$ at night).

\section{Initial Treatment}

Intravenous corticosteroids were insufficiently effective. Similarly, intrathecal triamcinolone only decreased spasticity for the first 4 weeks and therefore it was insufficiently effective.

Who Benefits Most from THC:CBD Spray?
Addition of THC:CBD Oromucosal Spray and Clinical Outcome

THC:CBD oromucosal spray (up-titrated to 3 sprays in the morning and 5 sprays at night) was started and the patient regained the ability to stand and transfer was possible with assistance. At a follow-up in June 2013 (after 611 days' treatment) the patient had improved motor function (EDSS 7.5). Treatment comprised THC:CBD oromucosal spray 3 sprays at night, baclofen $10 \mathrm{mg}$ at night and pregabalin (100 $\mathrm{mg}$ in the morning and $300 \mathrm{mg}$ at night) and was able to walk $4 \mathrm{~m}$ in $2 \mathrm{~min}$. Unaided transfer was also possible.

\section{Clinical Scenario 3: MS Patients with Relevant Impairment in Quality of Life/Activities of Daily Living (QoL/ADL)}

Worsening MS spasticity correlates with impairment of daily living and decreased QoL; it is also associated with increased disability which places a large burden on healthcare systems and costs [12-14]. THC:CBD oromucosal spray produced significant improvement in Barthel Activities of Daily Living scores compared to placebo ( $p=0.0067)$ in the largest Phase III study [1]. Furthermore, increases in QoL were reported by both patients and carers in a questionnaire survey of THC:CBD spray users in the UK [15].

\section{Case 5: Patient Details}

This case involved a 41-year-old female with secondary progressive MS, and an EDSS of 6.5. In 2001 the first incidence of MS (dysarthria, right-sided paresis and gait problems) was documented. Since 2011 there has been a 
progressive decrease in walking ability and in 2012 the patient developed recurrent temporary spasticity attacks with supination of the right foot. Regular antispasticity medication included baclofen $10 \mathrm{mg}$ three times a day and gabapentin (300 $\mathrm{mg}$ three times a day and $800 \mathrm{mg}$ at night). The walking distance was $58 \mathrm{~m}$ in $6 \mathrm{~min}$ with a walking frame.

\section{Initial Treatment}

Firstly, baclofen dosage was increased but this was ineffective and increased weakness. Increasing the dosage of gabapentin was also ineffective.

\section{Addition of THC:CBD Oromucosal Spray and Clinical Outcome}

THC:CBD oromucosal spray 3 sprays/day was added to the previous antispasticity regimen and there was an immediate improvement in walking distance. At a follow-up in June 2013 the patient was receiving baclofen 2.5 $\mathrm{mg}$ three times a day and gabapentin (300 $\mathrm{mg}$ three times a day and $800 \mathrm{mg}$ at night) plus THC:CBD oromucosal spray 3 sprays/day and the walking distance had improved to $160 \mathrm{~m}$ in $10 \mathrm{~min}$ with a walking frame and peroneal splint. QoL was improved for this patient since the increased mobility and ability to walk enabled her to go back to work.

\section{Case 6: Patient Details}

This case involved a 69-year-old male with secondary progressive MS, and an EDSS of 8.5. In 1978 the first incidence of MS (gait disturbances) occurred. From 2004 onwards there has been progressive weakness in the arms and legs and since 2012 there has been an increasing incidence of painful flexor spasticity attacks in the legs. The patient had trunk instability and movement was only possible with assistance. He was receiving baclofen $5 \mathrm{mg}$ three times a day and $10 \mathrm{mg}$ at night.

\section{Initial Treatment}

The baclofen dosage was decreased to improve tolerability while stabilizing the trunk but this increased flexor spasticity attacks. and there was an improvement in trunk stability and spasticity was decreased (NRS $8 / 10$ to $1 / 10$ ). Furthermore, transfer was easier and baclofen dosage was decreased to $5 \mathrm{mg} /$ day. At a follow-up in June 2013 (after

425 days treatment) the patient had maintained stable improvement in NRS scores. Treatment comprised THC:CBD oromucosal spray 5 sprays/day and pregabalin (50 $\mathrm{mg}$ in the morning and $75 \mathrm{mg}$ at night). Baclofen was discontinued. Inclusion of THC:CBD oromucosal spray in the treatment regimen resulted in meaningful improvement in condition and better sleep at night. This resulted in less fatigue during the day and greater ability to engage in physical and social activities.

\section{Conclusions}

From the outcomes of the treatment of this small sample of different MS spasticity typologies, it can be said that in clinical practice THC:CBD oromucosal spray is an effective and viable option for moderate to severe spasticity MS patients resistant to existing therapies. For some patients the dosage of existing therapies might be reduced and this can help minimise the impact of weakness and also limit the possibility of developing adverse drug effects. As observed in some clinical trials, relevant improvementsin QoL/ADL can be achieved using THC:CBD spray in patients with MS spasticity allowing them to engage in everyday activities with family, friends and work colleagues.

\section{Disclosure Statement}

J. Koehler received an honorarium from Laboratorios Almirall, SA, for his participation in the Symposium and producing his article, which is included in this Supplement. He has no other relevant affiliations or financial involvement with any organization or entity with a financial interest in or financial conflict with the subject matter or materials discussed in the manuscript apart from those disclosed. Writing assistance was provided by Content Ed Net, with funding from Laboratorios Almirall, SA.

\section{Addition of THC:CBD Oromucosal Spray and Clinical Outcome}

THC:CBD oromucosal spray 5 sprays/day was started

\section{References}

14
Novotna A, Mares J, Ratcliffe S, et al: A randomized, double-blind, placebo-controlled, parallel-group, enriched-design study of nabiximols* $\left(\right.$ Sativex $\left.^{\circledR}\right)$, as add-on therapy, in subjects with refractory spasticity caused by multiple sclerosis. Eur J Neurol 2011;18: 1122-1131.

-2 Wade D, Makela P, Robson P, House H, Bateman C: Do cannabis-based medicinal extracts have general or specific effects on symptoms in multiple sclerosis? A double-blind, randomized, placebo-controlled study on $160 \mathrm{pa}-$ tients. Mult Scler 2004;10:434-441. 
3 Wade DT, Makela PM, House H, Bateman C, Robson P: Long-term use of a cannabis-based medicine in the treatment of spasticity and other symptoms in multiple sclerosis. Mult Scler 2006;12:639-645.

-4 Collin C, Davies P, Mutiboko IK, Ratcliffe S, for the Sativex Spasticity in MS Study Group. Randomized controlled trial of cannabisbased medicine in spasticity caused by multiple sclerosis. Eur J Neurol 2007;14:290-296.

5 Collin C, Ehler E, Waberzinek G, Alsindi Z, Davies P, Powell K, et al: A double-blind, randomized, placebo-controlled, parallel-group study of Sativex, in subjects with symptoms of spasticity due to multiple sclerosis. Neurol Res 2010;32:451-459.

6 Montalbán X, Sastre Garriga J, Vila C, Clissold SP: THC and CBD oromucosal spray (Sativex) in the management of spasticity associated with multiple sclerosis. Expert Rev Neurotherapeutics 2011;11:627-637.
7 Robson P: Abuse potential and psychoactive effects of $\delta$-9-tetrahydrocannabinol and cannabidiol oromucosal spray (Sativex), a new cannabinoid medicine. Expert Opin Drug Saf 2011;10:675-685.

-8 García-Merino A: Endocannabinoid system modulator use in everyday clinical practice in the UK and Spain. Expert Rev Neurother 2013;13:9-13.

9 Flachenecker P: A new multiple sclerosis spasticity treatment option: effect in everyday clinical practice and cost-effectiveness in Germany. Expert Rev Neurother 2013;13(suppl 1):15-19.

10 Eltayb A, Etges T, Wright S: An observational post approval registry study of patients prescribed Sativex ${ }^{\circledR}$. Results from clinical practice. Presented at ECTRIMS 2013.
1 Shakespeare DT, Boggild M, Young C: Antispasticity agents for multiple sclerosis. Cochrane Database Syst Rev 2003;4:CD001332.

12 Flachenecker P, Zettl U, Essner U, Henze T: Drug treatment of spasticity in multiple sclerosis - a cross-sectional survey in Germany. Mult Scler 2012;18(4 suppl):478.

13 Oreja-Guevara C, González-Segura D, Vila C: Spasticity in multiple sclerosis: results of a Spanish patient survey. Int J Neurosci 2012; 123:400-408.

14 Arroyo R, Vila C, Clissold S: Retrospective observational study of the management of multiple sclerosis patients with resistant spasticity in Spain: the '5E' study. Expert Rev Pharmacoeconomics Outcomes Res 2011;11: 205-213.

15 Notcutt WG: A questionnaire survey of patients and carers of patients prescribed Sativex as an unlicensed medicine. Prim Health Care Res Dev 2013;14:192-199. 